\title{
APROXIMACIÓN AL DISEÑO DE UN SISTEMA DE INFORMACIÓN GERENCIAL PARA EL SECTOR ESTATAL EN COLOMBIA
}

\author{
Mauricio A. Cruz Pulido'
}

Recibido: 27 de abril de 2013

Aceptado: 12 de agosto de 2013

\section{Resumen}

El artículo presenta una propuesta de intervención mediante un sistema de información gerencial para el Estado Colombiano. Para tal propósito se presentan cuatro (4) secciones que describen de forma lógica el desarrollo del tema.

En la primera sección se establece el contexto estatal y se define el uso que el Estado da a la Tecnología de Información. La segunda parte del artículo presenta la propuesta de arquitectura empresarial AE para una entidad estatal y su relación con la agilidad. La tercera sección muestra la propuesta de diseño de la $A E$ con base a la investigación científica y finalmente la última sección establece los medios para medir la aceptación y utilidad de la propuesta de sistema de información.

Palabras clave: Nueva gestión pública, TIC, estrategia, arquitectura empresarial, investigación basada en el diseño, sistemas de información.

\section{APPROACH TO THE DESIGN OF A MANAGEMENT INFORMATION SYSTEM FOR GOVERNMENT IN COLOMBIA}

\section{Abstract}

The article presents a proposal for intervention by a management information system for the Colombian State. For this purpose there are four (4) sections describing a logical development of the subject. The first section sets the context state, and is defined using the state gives to IT. The second part of the paper presents the AE enterprise architecture proposal for a state agency and its relationship with the agility. The third section shows the proposed design of the $A E$ based on scientific research and finally the last section provides the means to measure the acceptance and utility of the proposed information system.

Keywords: New public management, TIC's, strategy, enterprise architecture, design-based research, information systems

\footnotetext{
${ }^{1}$ Candidato a Ph.D en Gestión y Ciencias Empresariales Universidad EAN-Universidad de Nebrija (España). 


\section{Introducción}

El presente artículo se fundamenta en la necesidad de fortalecer la capacidad de gestión del Estado en Colombia. Para ello parte de los esfuerzos hechos por el gobierno colombiano en relación con la política estatal de gobierno en línea y las bondades de las TIC en la modernización institucional y su impacto social relacionado con el mejoramiento de la gobernabilidad y la democratización de lo público.

El objetivo de la reflexión se orienta en articular una propuesta de diseño de sistema de información gerencial desde la arquitectura empresarial del sector público recogiendo la teoría de la racionalidad en la toma de decisiones propuesta y la investigación basada en el diseño bajo el enfoque de Simon (1967). Desde este punto de vista se abordan los conceptos y elementos de la misma evidenciando los beneficios en términos de eficiencia y agilidad para el Estado en Colombia.

Finalmente se propone un instrumento que permite medir el impacto en la gestión de organizaciones públicas que apliquen modelos de arquitectura empresarial y sistemas de información gerencial basados en el uso de las tecnologías de información y comunicaciones TIC.

\section{TIC en la práctica en el Estado colombiano}

El Estado y su gerencia se ve inmerso en una nueva realidad organizacional vinculada transversalmente por los postulados del new public management con lo cual se ve obligado a modernizar su estructura con el objetivo de prestar servicios con mayor calidad, eficiencia y eficacia. Al respecto Holmes (2003) plantea que los gobiernos que se conformen con las antiguas formas de hacer negocios quedarán expuestos al ridículo y a acusaciones de incompetencia y de corrupción. En este sentido los ciudadanos esperan de las organizaciones estatales la oferta de servicios en línea como consecuencia de la estandarización y normalización de sus procesos, procedimientos, planes, programas y proyectos.
La nueva gestión pública y el uso de las TIC generan una sinergia fundamental para administrar, gerenciar, controlar, proveer e innovar en la prestación de servicios que permitan que los objetivos del desarrollo socio-económico se logren efectivamente. De esta forma el Estado debe evolucionar con el soporte de una gestión estratégica mediada por las TIC, que tal como lo plantea Barzelay (2003), se enmarca en un proceso de argumentación acerca de cómo se gerencian las organizaciones complejas.

En este sentido conceptos como democracia, gobernabilidad, gobernanza y buen gobierno son referentes de la modernización del Estado, proceso en el que las TIC juegan un papel protagónico.

\section{Modernización estatal y TIC en la práctica}

Dado el proceso de modernización el Estado colombiano ha puesto en marcha la estrategia de Gobierno en Línea GEL la cual se enmarca como uso de las TIC en la práctica.

La política de Gobierno en línea pretende la construcción de un Estadomás eficiente, más transparente y participativo, y que presta mejores servicios con la colaboración de toda la sociedad, mediante el aprovechamiento de las TIC, de tal forma que por este medio se logre la difusión de información, el facilitar proveer trámites y servicios en línea, así como el fortalecimiento de la democracia por medios electrónicos (Barzelay, 2003).

Para el cumplimiento de esta política, el gobierno nacional se ha alineado con las tendencias y estándares internacionales, con la normatividad actual y con las necesidades y servicios de cada una de las Entidades oficiales, de tal forma que la implementación de esta política realmente favorezca a los ciudadanos, empresarios y servidores públicos. 
A través de la estrategia GEL el Estado Colombiano busca además incursionar en el Open Government OGov o Gobierno Abierto como una nueva forma con la cual la administración pública busca relacionarse con los ciudadanos.

La estrategia GEL busca que cada una de las entidades del Estado estructuren sus procesos de forma sistémica de manera tal que se convierta en un sistema de información para la toma de decisiones a nivel interno y en un canal abierto de relación con el ciudadano.

De igual forma el Estado busca que las TIC sirvan como herramienta para crear nuevas estructuras organizativas fundamentadas en modelos en red, orientados a proyectos y al logro de resultados.

\section{Propuesta de arquitectura empresarial}

Las TIC en el Estado colombiano son un componente que coadyuva a generar nuevas formas de gobernar lo que induce a construir distintas estrategias que conduzcan a innovadores tipos de transacciones y servicios, a otras maneras de relacionarse con el ciudadano y a otros caminos de organización y gestión de la información.

Lo anterior se debe soportar en una arquitectura empresarial o institucional, que de acuerdo con Rivera (2011) se concibe como una herramienta estratégica que provee una estructura adecuada, funcional y dinámica en la cual se deben enmarcar los sistemas de información que se construyan para el apoyo a las entidades en el cumplimiento de sus objetivos misionales.

La arquitectura empresarial (AE), como enfoque estratégico describe la forma en que la institución organiza su infraestructura de tecnologías de información TI, identifica las capacidades de procesos de negocio para hacer frente a sus necesidades de $\mathrm{Tl}$ e integra y normaliza dichos procesos. La arquitectura empresarial (AE) sustenta las decisiones relativas a los datos, aplicaciones infraestructura de TI (técnicos y humanos) y la gestión de responsabilidades. La $\mathrm{AE}$ es una guía para la implementación de TI y una hoja de ruta a los futuros recursos de TI en la organización (Bradley et al. 2011).

Como práctica o forma de trabajar, la $\mathrm{AE}$ se basa en un conjunto de principios de visión sistémica, mejora continua e identificación integral de todos los impactos que tendría la organización en caso de ser necesario un ajuste de operación (Hernández, 2008).

\section{AE y su contribución a la agilidad estatal}

Las entidades estatales deben implementar sistemas de información soportados en arquitecturas institucionales que gestionen o agilicen la información y soporten los procesos de planeación, ejecución, medición y control. El contexto y el quehacer estatal tienen una dependencia con la ley, su implementación y la tecnología que permite su aplicación.

De esta forma la AE permite a las instituciones estatales interpretar y ejecutar el marco jurídico y normativo que delinea su misión y visión organizacional. La normalización y estandarización de procesos, procedimientos, planes, programas y proyectos hacen menos complejas las actuaciones y toma de decisiones de los gerentes públicos (Rivera, 2011).

La AE maximiza y representa la generación de valor en los servicios que el Estado presta a ciudadanos, empresarios y otras instituciones. En este sentido se expresa una mejora cualitativa y cuantitativa de la gestión pública en términos de calidad, celeridad y eficiencia en la prestación de servicios y transparencia de lo público. De la misma manera hace a los gobiernos más democráticos, participativos, competitivos y productivos beneficiando la calidad de vida de sus ciudadanos. 
El Estado se beneficia con la $\mathrm{AE}$ debido a que adquiere la capacidad de adelantarse a las necesidades del ciudadano mediante la continua innovación en sus procesos, procedimientos y servicios además permite al management público tener una visión holística de la organización estatal permitiéndole tomar decisiones acertadas en un contexto coyuntural.

En términos financieros la implementación de arquitecturas institucionales reduce los costos de operación y producción de servicios por parte del Estado beneficiando la disminución del déficit fiscal.

\section{Método de diseño participativo para construir la arquitectura empresarial}

De acuerdo con González (2007) la investigación científica basada en el diseño designa conocimientos específicos que son elaborados para resolver, de manera articulada, problemas concretos que surgen en el entorno humano. Este nuevo campo del saber, de carácter artificial, abarca además un conjunto de prácticas científicas propias que son diferentes de las utilizadas en otras ciencias empíricas (González, 2007). Niiniluoto (1995), señala que el diseño científico es la actividad de solucionar problemas mediante el uso de métodos científicos y conocimiento científico

De acuerdo con González (2007) la investigación científica basada en el diseño es el resultado de la «cientificación» del arte, la tecnología, la gestión o el desarrollo. De esta manera habría primero una actividad profesional relacionada por ejemplo con el management, para llegar después a la elaboración de un diseño científico (Niniluoto, 1995).

La propuesta de Simón (1976) se orienta a que el diseño como ciencia es un instrumento tanto para comprender como para actuar, mediante un proceso de entender mejor la información disponible y actuar a partir de ella. El mismo autor enlaza las «ciencias del diseño» con las "ciencias de lo artificial» y en este sentido señala que en las ciencias del diseño existe una creatividad humana de fondo, que modula los sucesivos pasos como ciencias de lo artificial: los objetivos, los procesos y los resultados.

Plantea que el diseño establece metas posibles y alcanzables, de tal forma que mediante unos procesos bien articulados puede llevar al resultado anticipado. De acuerdo con Simón (1976) las ciencias del diseño versan sobre un mundo construido y además, lo intentan ampliar mediante predicciones (anticipación del futuro) y prescripciones (pautas de actuación para resolver problemas).

Simón (1976), asigna el «crear lo artificial» a las «ciencias del diseño» lo que requiere racionalidad tanto en el plano de la actividad científica en sí misma como en el ámbito de los agentes que la originan. De este modo el diseño se enraíza en la racionalidad de los agentes que el autor concibe como «racionalidad limitada».

\section{Diseño participativo}

Desde la perspectiva de la racionalidad limitada los agentes construyen sus diseños: buscan unos objetivos, seleccionan unos procesos y esperan unos resultados. Las predicciones que realizan y las prescripciones que hacen para alcanzar las metas trazadas se apoyan en un tipo de conocimiento y en una capacidad de computación que son limitados. Según Ninniluoto (1995) los agentes se encaminan a la resolución de problemas concretos, para lo que suelen contar con una práctica profesional previa.

González (2012) señala la necesidad de la participación en el diseño lo que requiere la coordinación de conocimientos, recursos, objetivos y requerimientos de distintos actores. Dichos actores no solo proveen requerimientos, sino también evalúan el resultado. Así las cosas el diseño es un procesos distribuido y multiagente. 
El diseño participativo parte de las propias creencias y visiones de los agentes lo cual ante una serie de objetivos les lleva a actuar de determinadas formas. Dichas acciones que afectan o perturban el mundo real producen una serie de efectos que pueden ser los deseados o de forma contraria indeseados. El proceso se retroalimenta constantemente y el aprendizaje continuo hace que cada vez se minimice el error (González, 2012).

De acuerdo con lo anterior se establece la propuesta de método de diseño participativo para la construcción de la arquitectura empresarial en una entidad del Estado colombiano.

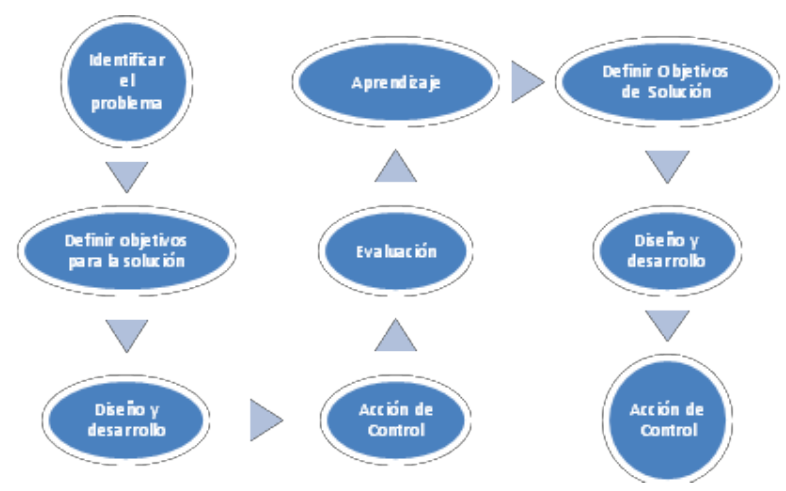

Fuente: Propia adaptada de González (2012).

Análisis del Entorno: el diagnostico participativo del entorno incluye la información tomada de todos los actores como lo son las personas, la tecnología y la organización.

Identificación del problema: el diagnostico en el cual participan diversos actores permite identificar una situación problemica que para el caso es: complejidad en la toma de decisiones y actuaciones de los gerentes públicos.

Objetivos para la solución: la definición del problema conduce a plantear su solución mediante el planteamiento del objetivo que para el caso es: normalización y estandarización de procesos, procedimientos, planes, programas y proyectos en la entidad estatal.
Diseño y desarrollo: la siguiente etapa consiste en la construcción del artefacto el cual deberá ser riguroso mediante un proceso de investigación científica multi- agente que para el caso es el sistema de información soportado en una arquitectura empresarial que gestione los procesos de planeación, ejecución, medición y control de la entidad estatal. Acción de Control: en esta etapa se pone a prueba el artefacto por parte del gerente público

Evaluación: la puesta en práctica del artefacto evidenciará su eficiencia o eficacia para solucionar la problemática definida por los actores en el diagnostico participativo.

Aprendizaje: el proceso de retroalimentación concluirá la racionalidad del artefacto. Si los resultados no son los esperados existirá la posibilidad del rediseño hasta que el error sea mínimo y la problemática sea solucionada.

\section{Aceptación y éxito de la propuesta de arquitectura empresarial}

Diversos modelos son usados para medir la aceptación de la tecnología de información TI Davis (1989) propone el Technology Acceptance Model TAM con efectivos resultados como medio para predecir el uso de la TI.

EI TAM se basa en dos características principales: (i) La utilidad percibida y (ii) la facilidad de uso percibida.

La utilidad percibida hace referencia al grado en que una persona cree que usando determinado sistema mejorará su desempeño en el trabajo. La facilidad de uso percibida muestra hasta qué grado una persona cree que usando un sistema realizará menos esfuerzo desempeñando sus tareas ( Yong, 2004 ).

De acuerdo a este modelo las percepciones del individuo sobre la utilidad y la facilidad de uso 
son determinantes al momento de predecir la intención de usar el sistema de información.

Yong (2004) señala que los modelos de aceptación de sistemas de información incluyen variables externas que tienen influencia indirecta en la actitud hacia usar, intención conductual para usar y la conducta de uso real. González (2012) establece que la mayoría de los modelos han sido revisados con la prueba de variables moderadoras como género, edad, volición y experiencia, las cuales influyen en la aceptación del sistema.

Para el caso de estudio se propone que el modelo de aceptación tecnológica se fundamente en una investigación de campo que permita recolectar información mediante un instrumento de encuesta cara a cara.

El instrumento de investigación (encuesta) se dividirá en dos secciones, la primera con una serie de preguntas en escala de likert que permitan medir la intención y facilidad de uso del sistema de información. La segunda parte se diseñará para obtener información acerca de datos demográficos como género, edad, nivel de educación, cargo y dependencia a la que pertenece en la entidad.

\section{Utilidad potencial del sistema de información}

Los modelos de éxito y que miden la utilidad potencial de los sistemas de información se fundamentan en la propuesta de Delone y McLean (1992) con lo cual el análisis para nuestro caso debe tener las siguientes dimensiones: calidad del sistema, calidad de la información, calidad del servicio y beneficios netos.

Calidad del Sistema: Se centra en las características del sistema de procesamiento de información en sí mismo (Delone y McLean, 1992).
Para nuestro caso se debe medir el grado de productividad, portabilidad, fiabilidad y facilidad de uso del sistema de información para la entidad pública.

Calidad de la información: se centra en las características de la información que produce el sistema primariamente en forma de informes 0 reportes. Para nuestro caso y de acuerdo con Yong (2004) este aspecto debe evaluar si la información que reporta el sistema es utilizable, concisa, comprensible y pertinente.

Calidad de servicio: Esta dimensión captura la calidad de servicio que la función de sistemas de información otorga a la organización (Delone y McLean, 1992). Para el caso se relaciona con evaluación de factores como tangibilidad, fiabilidad,capacidad de respuesta y seguridad del sistema de información gerencial para la entidad pública.

Beneficios netos: Esta dimensión mide los efectos positivos del sistema de información (Delone y Mclean, 1992). Indican que cada estudio debe definir el contexto en el cual se darán esos beneficios, es decir, quién o quiénes serán los beneficiarios. Para el caso el beneficio neto es la entidad pública y los beneficiarios los gerentes públicos quienes verán reducida la complejidad a la hora de tomar decisiones.

De acuerdo a lo anterior se evidencia que la utilidad del sistema de información se mide mediante una epistemología pragmática a través de la investigación basada en el diseño. El punto de partida es la formulación del problema hasta medir si el diseño cumple o no con la solución de la problemática. 


\section{Conclusiones}

El Estado Colombiano debe considerar el uso de la TI como una práctica que le permitirá ser más eficiente, eficaz, productivo y competitivo en busca del objetivo de tomar mejores decisiones que impacten la calidad y el bienestar de los ciudadanos.

Dentro del proceso de Modernización Estatal es un imperativo que los gobiernos adopten el uso de las TI soportados en arquitecturas institucionales o empresariales que le permitan una mayor agilidad en términos del desarrollo de sus procesos internos, atención al ciudadano y calidad del servicio.

La investigación científica basada en el diseño toma información relevante del entorno y proveniente de distintos actores. La construcción de artefactos, modelos, métodos o teorías son consecuencia del diseño y de la rigurosidad del proceso de investigación.

El diseño participativo en el sector estatal debe ser una característica fundamental del proceso de investigación ya que favorece el aprendizaje y la retroalimentación de resultados.

Los modelos de aceptación y medición de sistemas de información se fundamentan en epistemologías investigativas que permiten predecir su uso y medir su utilidad potencial.

El e-government como plataforma estructurada de arquitectura institucional es el medio con el cual los gobiernos de economías emergentes pueden mejorar su capacidad de gobierno y con ello mejorar de forma sustancial la calidad en la prestación de servicios a la comunidad sin importar la ubicación o la demanda del ciudadano.

\section{Bibliografia}

Akkermans, H.; Van Helden, K. (2002). Vicious and virtuous cycles in ERP implementation: a case study of interrelations between critical success factors, European Journal of Information Systems, 11 (1): 35-46.

Barzelay, M. (2003). The new public management. Berkeley: University of California Press.

Bradley, Et al. (2011). The Role of Entreprise Architecture In The Quest for It Value. MIS Quarterly Executive. Vol 10. No.2.

Delone, W., Mclean, E. (1992). The Delone and McLean Model of Information Systems Success. Informations Systems Research, 3: 60-95.

Delone,W., Mclean, E. (2008). Measuring information systems success: models, dimensions, measures, and interrelationships. European Journal of Information Systems, 17(3): 236-263.

Escobar, B.; Rocha, C. M. (2003). La investigación en sistemas integrados de gestión: Una revisión de las principales revistas españolas. ponencia presentada en XIII Jornadas Hispano-Lusas de gestión cientifica.

Gonzalez, R. A. \& Sol, H.G. (2012). Validation and Design Science Research in Information Systems in M. Mora, O. Gelman, A.L. Steenkamp \& M. Raisinghani (Eds.)

Hernández. E. (2008). Arquitectura Empresarial como práctica para mantener la estabilidad de los sistemas en una organización. Disponible en: http://www.repositoriodigital.ipn.mx/bitstream/handle/123456789/5392/47-3. pdf? sequence $=3$

Holmes, D. (2003). Estrategias de negocios electrónicos para el gobierno. OEA.

Rivera, G. (2011). Arquitectura Empresarial como herramienta de soporte estratégico a las entidades del Estado. Research Methodologies, Innovations and Philosophies in Software Systems Engineerin and Information Systems. Hershey: IGI Global.

Simon, H. (1976). The Sciences of the artificial. New Jersey: Princeton University.

Yong. L. (2004). Modelo de Aceptación Tecnológica TAM para determinar los efectos de las dimensiones de cultura nacional en la aceptación de las TIC. Revista Internacional de Ciencias Sociales y Humanidades, enerojunio, año/vol. XIV (1): 131-171.

Zhang, L.; Lee, M.; Zhang, Z.; Banerjee P. (2003). Critical Success Factors of Enterprise Resource Planning Systems Implementation Success in China, Proceedings of the 36th Hawaii International Conference on System Sciences. 


\section{Anexo}

Variable:

Utilidad Percibida: grado en que una persona cree que usando un sistema en particular mejorará su desempeño en el trabajo

Indicador: Índice Alto=muy útil

\section{Características:}

- Uso Frecuente del sistema en particular por el gerente

- Alta eficiencia y eficacia en el trabajo del usuario

- Alta Gerencia

Indicador: Índice Bajo= No útil

\section{Características:}

- Uso moderado o bajo de un sistema por el gerente público

- Baja eficiencia y eficacia en el trabajo del usuario

- Nivel medio de la organización

\section{Instrumento}

1. El sistema de información me permite que tome mejores decisiones

2. Usando el sistema de información mejora mi productividad

3. Usando el sistema de información mejora la efectividad en mi trabajo

4. Usando el sistema de información se hace más fácil tomar decisiones

5. En conjunto, yo encuentro que un sistema de información es útil para tomar decisiones

\section{Escala tipo Likert de 5 puntos}

\section{Variable}

Facilidad de Uso Percibida: Señala hasta que grado una persona cree que usando un sistema en particular realizará menos esfuerzo para desempeñar sus tareas

Indicador: Índice Alto= Muy fácil

\section{Características:}

- Mejor rendimiento en el trabajo del gerente público

- Alta satisfacción laboral del gerente

- Utilización alta del sistema de información

Indicador: Índice Bajo=Difícil

Características:

- Menor rendimiento en el trabajo del gerente público

- Baja satisfacción laboral

- Utilización baja del sistema de información

\section{Instrumento}

1. Aprender a operar el sistema de información es fácil para mí

2. Es para mi fácil entender el sistema de información y usarlo para lo que yo quiero

3. Mi interacción con el sistema de información es clara y entendible

4. En conjunto considero que el sistema de información es fácil de usar

\section{Escala de tipo Likert 5 puntos}

